\title{
Response: Limited sampling strategies for once daily tacrolimus exposure monitoring
}

\author{
D. J. A. R Moes ${ }^{1}$ - J. J. Swen ${ }^{1}$ - S. A. S van der Bent ${ }^{2}$ - T. van der Straaten ${ }^{1}$. \\ A. Inderson ${ }^{2}$ - E. Olofsen ${ }^{3}$ - H. W. Verspaget ${ }^{2}$ - H. J. Guchelaar ${ }^{1}$ • J. den Hartigh ${ }^{1}$. \\ B. van Hoek ${ }^{2}$
}

Received: 24 February 2016 / Accepted: 25 February 2016 /Published online: 2 March 2016

(C) Springer-Verlag Berlin Heidelberg 2016

Dear Sir,

We thank Dr. Antonio J. Carcas-Sansuán for his valuable comments. However, there is an important difference between the Madrid models and the Leiden models. In their article (Almeida-Paulo et al.) [1], linear regression limited sampling strategies for $\mathrm{AUC}_{12}$ prediction were developed and not limited sampling maximum a priori (MAP) Bayesian estimation models. Linear regression limited sampling strategies are inconvenient in a way that it requires strict adherence to set times for blood sample collection which is almost impossible in clinical practice. Erroneous predictions will occur if the sampling times are not exactly as requested in the sampling protocol. In contrast, maximum a priori (MAP) Bayesian population pharmacokinetic models as we have developed in our recent article [2] are far more flexible and can handle sampling times which deviate from the sampling protocol without resulting in erroneous predictions. Although these models require more complex calculations and the development of a population pharmacokinetic model, we believe these models are superior to linear regression limited sampling formulas

D. J. A. R Moes

d.j.a.r.moes@lumc.nl

1 Department of Clinical Pharmacy and Toxicology, Leiden University Medical Center, Leiden, The Netherlands

2 Department of Gastroenterology and Hepatology, Leiden University Medical Center, Leiden, The Netherlands

3 Department of Anesthesiology, Leiden University Medical Center, Leiden, The Netherlands when used in clinical practice. Currently different software packages are available to support these population phamacokinetic models in clinical practice and have been evaluated recently [3]. Furthermore, different more user friendly web-based initiatives are emerging such as Dose $\mathrm{Me}$ [4] and Insight-rx [5]. In our University Medical Center, we have used these kinds of models for over a decade in routine clinical practice for prediction of cyclosporine, tacrolimus, and mycophenolic acid exposure for all renal and liver transplant recipients with satisfactory results. Furthermore, Størset et al. $[6,7]$ recently showed that computerized dose individualization improved target concentration achievement of tacrolimus after renal transplantation potentially improve long-term outcome. We agree with Dr. Antonio J. Carcas-Sansuán that hard evidence that $\mathrm{AUC}_{12}$ monitoring of tacrolimus is superior to trough monitoring is still lacking when looking at clinical endpoints. However, based on theory and examples from clinical practice, we strongly believe that $\mathrm{AUC}_{12}$ monitoring of tacrolimus is more informative and accurate and should therefore be applied. The development of dried blood spot techniques $[8,9]$ has also made the limited sampling $\mathrm{AUC}_{12}$ for patient less burdensome because the blood sampling can now be performed at home. To gain more evidence with respect to improvement of clinical outcome using these models, we would suggest to set up a two arm randomized clinical trial in a high acute rejection risk patient population to evaluate the differences in acute rejection episodes and renal function decline over a period of for instance 5 years between the trough monitoring arm and the limited sampling $\mathrm{AUC}_{12}$ monitoring arm. Such a study would be able to provide indisputable evidence for potential additional value of $\mathrm{AUC}_{12}$ over trough monitoring; however, it will be challenging to finance such a study with a high sample size and long-term follow-up. 


\section{References}

1. Almeida-Paulo GN, Lubomirov R, Alonso-Sanchez NL et al (2014) Limited sampling strategies for tacrolimus exposure (AUC0-24) prediction after Prograf ${ }^{\mathbb{B}}$ and Advagraf ${ }^{\mathbb{B}}$ administration in children and adolescents with liver or kidney transplants. Transpl Int 27(9):939-948

2. Moes DJAR, van der Bent SAS, Swen JJ et al (2015) Population pharmacokinetics and pharmacogenetics of once daily tacrolimus formulation in stable liver transplant recipients. Eur J Clin Pharmacol 31(4):223-224

3. Fuchs A, Csajka C, Thoma Y, Buclin T, Widmer N (2013) Benchmarking therapeutic drug monitoring software: a review of available computer tools. Clin Pharmacokinet 52(1):9-22

4. Dose Me (2016) Dose me. Available from: https://doseme.com.au/. Accessed 24 Feb 2016

5. Insight-rx (2016) Insight-rx. Available from: http://www.insight-rx. $\mathrm{com} /$. Accessed 24 Feb 2016
6. Størset E, Holford N, Hennig S et al (2014) Improved prediction of tacrolimus concentrations early after kidney transplantation using theory-based pharmacokinetic modelling. Br J Clin Pharmacol 78(3):509-523

7. Størset E, Åsberg A, Skauby M et al (2015) Improved tacrolimus target concentration achievement using computerized dosing in renal transplant recipients - a prospective, randomized study. Transplantation 99(10):2158-2166

8. Koster RA, Greijdanus B, Alffenaar J-WC, Touw DJ (2015) Dried blood spot analysis of creatinine with LC-MS/MS in addition to immunosuppressants analysis. Anal Bioanal Chem 407(6):15851594

9. Koster R, Alffenaar JWC, Greijdanus B, Uges DR (2013) Fast LC$\mathrm{MS} / \mathrm{MS}$ analysis of tacrolimus, sirolimus, everolimus and cyclosporin $\mathrm{A}$ in dried blood spots and the influence of the hematocrit and immunosuppressant concentration on recovery. Talanta 115:47-54 